\title{
Altered IL-32 Signaling in Abdominal Aortic Aneurysm
}

\author{
Sophy Bengts ${ }^{a}$ Levar Shamoun ${ }^{b, c}$ Anne Kunath ${ }^{c}$ Daniel Appelgren ${ }^{a}$ \\ Martin Welander ${ }^{d}$ Martin Björck ${ }^{\mathrm{e}}$ Anders Wanhainen ${ }^{\mathrm{e}}$ Dick Wågsäter ${ }^{\mathrm{a}, \mathrm{c}}$ \\ ${ }^{a}$ Division of Drug Research, Department of Medical and Health Sciences, Linköping University, Linköping, Sweden; \\ bDivision of Medical Diagnostics, Department of Laboratory Medicine, Jönköping County, Jönköping, Sweden; \\ 'Department of Medical Cell Biology, Uppsala University, Uppsala, Sweden; dDivision of Cardiovascular Medicine, \\ Department of Medical and Health Sciences, Linköping University, Linköping, Sweden; ${ }^{e}$ Department of Surgical \\ Sciences, Section of Vascular Surgery, Uppsala University, Uppsala, Sweden
}

\section{Keywords}

Abdominal aortic aneurysm - Inflammation - Leukocytes .

T cells $\cdot$ Cytokines

\begin{abstract}
Introduction and Objective: Interleukin (IL)-32 is a pro-inflammatory cytokine not previously studied in relation to abdominal aortic aneurysm (AAA). The aim of this study was to elucidate the expression and localization of IL-32 in AAA. Methods: Expression and localization of IL-32 in human aortic tissue was studied with immunohistochemical analysis and Western blot (AAA: $n=5$; controls: $n=4$ ). ELISA was used to measure IL-32 in human plasma samples (AAA: $n=140$; controls: $n=37$ ) and in media from cultured peripheral blood mononuclear cells (PBMCs) from 3 healthy donors. IL-32 mRNA in PBMCs, endothelial cells, aortic smooth muscle cells (SMCs), and aortic tissue samples of AAA $(n=16)$ and control aortas $(n=9)$ was measured with qPCR. Results: IL-32 was predominantly expressed in SMCs and T-cell-rich areas. Highest mRNA expression was observed in the intima/media layer of the AAA. A weaker protein expression was detected in non-aneurysmal aortas. Expression of IL-32 was confirmed
\end{abstract}

in isolated T cells, macrophages, endothelial cells, and SMCs, where expression was also inducible by cytokines such as interferon- $\gamma$. There was no difference in IL-32 expression in plasma between patients and controls. Conclusion: IL-32 signaling is altered locally in AAA and could potentially play an important role in aneurysm development. Further studies using animal models would be helpful to study its potential role in AAA disease.

(C) 2020 The Author(s) Published by S. Karger AG, Basel

\section{Introduction}

Abdominal aortic aneurysm (AAA) is a common and potentially lethal disease [1-4]. The causal events leading to the development of an AAA remain partially unknown, but the disease is a result of multiple genetic and environmental interactions [5]. The driving forces behind the progression of AAA expansion have been linked to the immune system. The chronic invasion of inflammatory cells into the vascular wall combined with the expression of local proteases leads to the loss of extracellular matrix. This contributes to the eventual irreversible weakening of 
the vascular wall [6-9]. The inflammatory cells referred to include macrophages, $\mathrm{CD} 4^{+} \mathrm{T}$ lymphocytes, neutrophils, B lymphocytes, mast cells, and natural killer cells $[6$, $10,11]$.

A study by Son and colleagues showed that interleukin-32 (IL-32, gene ID: 9235) inhibits endothelial inflammation, vascular smooth muscle cell (SMC) activation, and atherosclerosis [12]. First described as natural killer transcript 4 [13], the pro-inflammatory cytokine IL-32 induces the production of tumor necrosis factor (TNF)- $\alpha$ [14-16], IL-8, and macrophage inflammatory protein-1. IL-32 can act via classical pro-inflammatory pathways triggering nuclear factor- $\mathrm{\kappa B}$ and mitogen-activated protein kinase [15]. Through the amplification of responses induced by pro-inflammatory cytokines and chemokines, IL-32 promotes inflammatory status $[14,16]$. A mechanism by which the inflammatory status of vessels is amplified is via the synergizing of IL-32 and IL-1 $\beta$ [14]. A primary target for IL- $1 \beta$ is the vascular endothelium, where IL-1 $\beta$ can increase inflammation [17-19]. In an IL32 -dependent manner, IL-1 $\beta$ induces the upregulation of ICAM-1, an adhesion factor on endothelial cells, which increases the recruitment of leukocytes and the level of inflammation [20].

The synthesis of IL-32 is induced by pro-inflammatory cytokines, which includes IL- $1 \beta$, TNF- $\alpha$, and interferon (IFN) $-\gamma[15,21,22]$. Via the nuclear factor- $\kappa B$ pathway, IL- $1 \beta$, TNF- $\alpha$, and IFN- $\gamma$ activate the transcription of IL-32 mRNA in endothelial cells, macrophages, and monocytes [23]. The result of the synthesis of IL-32 is a propagated, positive loop leading to the increased production of TNF- $\alpha$, which in turn induces the synthesis of IL-32 [15, 21-23].

To date, the pathological mechanisms behind an AAA are not fully understood, but a possible pathway is mediated through chronic inflammation. To our knowledge, IL-32 has so far not been studied in relation to AAA disease. The aim of this study was to examine the expression, localization, and cells expressing IL-32 in the tissue of patients with AAAs.

\section{Materials and Methods}

\section{Patient Samples}

Samples were collected from individuals recruited for a screening study in southern Sweden. The inclusion criterion for AAA was initial abdominal aortic diameter $\geq 30 \mathrm{~mm}$. All patients were followed up prospectively from the time of enrolment into AAA surveillance. An overview of samples and methods used are described in online suppl. Tables 1 and 2 (for all online suppl. material, see www.karger.com/doi/10.1159/000507667).
Biopsies were fixed in $4 \%$ formaldehyde for immunohistological analyses, snap-frozen in liquid nitrogen for protein analysis, or placed in RNAlater (Ambion, Austin, TX, USA) overnight at $4^{\circ} \mathrm{C}$, and then stored at $-80^{\circ} \mathrm{C}$ for RNA isolation as described previously [24]. Tissue samples were from non-AAAs and AAAs (aortic diameter $>55 \mathrm{~mm}$ from computed tomography scan). Protein lysate samples used for Western blot included 4 control samples (all males, age $34 \pm 11.1$ years) and 5 AAA samples ( 1 female and 4 males, age $75 \pm 3.1$ years).

EDTA plasma samples procured for ELISA included 37 controls and 140 samples from AAA patients who were age and sex matched ( $70 \pm 2.5$ and $70 \pm 4.0$ years, respectively). Patient samples used for qPCR included cDNA isolated from 9 non-aneurysmal aortas ( 7 males and 2 females, age $45 \pm 13.4$ years) and 16 AAAs (14 males and 2 females, age $72 \pm 5.4$ years) that were also divided into layers of intima/media, adventitia, and perivascular aortic tissue (PVAT), where tissue was available.

All AAA participants gave written informed consent to the study, which was approved by the regional ethical review board in Linköping, Sweden. Control aortas without clinical or macroscopic signs of aortic atherosclerosis or aneurysm were obtained from organ donors collected from medicolegal autopsies, approved by the regional ethical review board in Lund, Sweden. The study protocol conforms to the ethical guidelines of the 1975 Declaration of Helsinki.

\section{Cell Cultures}

Human vascular endothelial cells (HUVECs: \#CC-2517) and aortic smooth muscle cells (AoSMCs: \#CC-2571) (Clonetics; Cambrex Bio Science Walkersville, Inc.; Lonza Group Ltd., Basel, Switzerland) were grown in recommended supplemented media (AoSMCs: \#CC-3182; HUVECs: \#CC-3162, Clonetics) and stimulated with similar concentrations of agents according to previous studies [20,25-27]. HUVECs were stimulated with a mix of TNF- $\alpha$ $(20 \mathrm{ng} / \mathrm{mL})$ and IL-1 $\beta(10 \mathrm{ng} / \mathrm{mL})$, or lipopolysaccharide (50 ng/ $\mathrm{mL})$ for 4,24 , and $48 \mathrm{~h}$. AoSMCs were stimulated with IFN- $\gamma(20$ $\mathrm{ng} / \mathrm{mL})$ for 4,24 , and $48 \mathrm{~h}$ or with TNF- $\alpha(20 \mathrm{ng} / \mathrm{mL})$ and IL- $1 \beta$ (10 ng/mL) for 4 and $24 \mathrm{~h}$.

This study includes qPCR of leukocytes from 3 healthy individuals (male; age 34,35 , and 38 years). Neutrophils were isolated through Percoll density centrifugation, whereas $\mathrm{T}$ cells, monocytes, and B cells were isolated through positive selection using magnetic-activated cell sorting (MACS, Milteniy Biotec, Germany), by using microbeads against CD3, CD14, and CD19, respectively. The cells were stimulated with IFN- $\gamma(20 \mathrm{ng} / \mathrm{mL})$ for $2 \mathrm{~h}$ (neutrophils) or $40 \mathrm{~h}$ (T cells, monocytes, and B cells). Unstimulated cells from the same individuals, isolated at the same time, acted as controls. All cells were cultured in $5 \% \mathrm{CO}_{2}$ at $37^{\circ} \mathrm{C}$, and unstimulated cells prepared at the same time acted as controls.

\section{Immunohistochemistry}

Samples were soaked for $20 \mathrm{~min}$ at $100^{\circ} \mathrm{C}$ in $5 \%$ Diva Decloaker (Biocare Medical, USA) for antigen retrieval. Three percent hydrogen peroxide solution was used to block the activity of endogenous peroxidase. Normal goat serum (20\%) was used for blocking. For the visualization of IL-32 in human samples, a rabbit polyclonal IL32 antibody (\#ab37158, Abcam, UK) was used, which is designed to detect all major isoforms, that is, $\alpha, \beta, \delta$, and $\gamma$. The antibody was used in a 1:300 dilution $(0.0033 \mu \mathrm{g} / \mu \mathrm{L})$ and applied to the samples for $60 \mathrm{~min}$ at room temperature. For staining of cell markers, a-actin (rabbit polyclonal, \#A5228, Sigma Aldrich, St. Louis, MO, 
Fig. 1. Representative immunohistochemical localization of IL-32 in the tunica adventitia of the aneurysmal tissue from a 65-year-old man with an aneurysm of $60 \mathrm{~mm}$. Expression of IL-32 (a, b), a-actin (c, d), von Willebrand (e, f), CD $3 \varepsilon(\mathbf{g}, \mathbf{h})$, and isotypic IgG control for IL-32 (i, j). Scale bar $=200 \mu \mathrm{m}$. IL-32, interleukin-32.

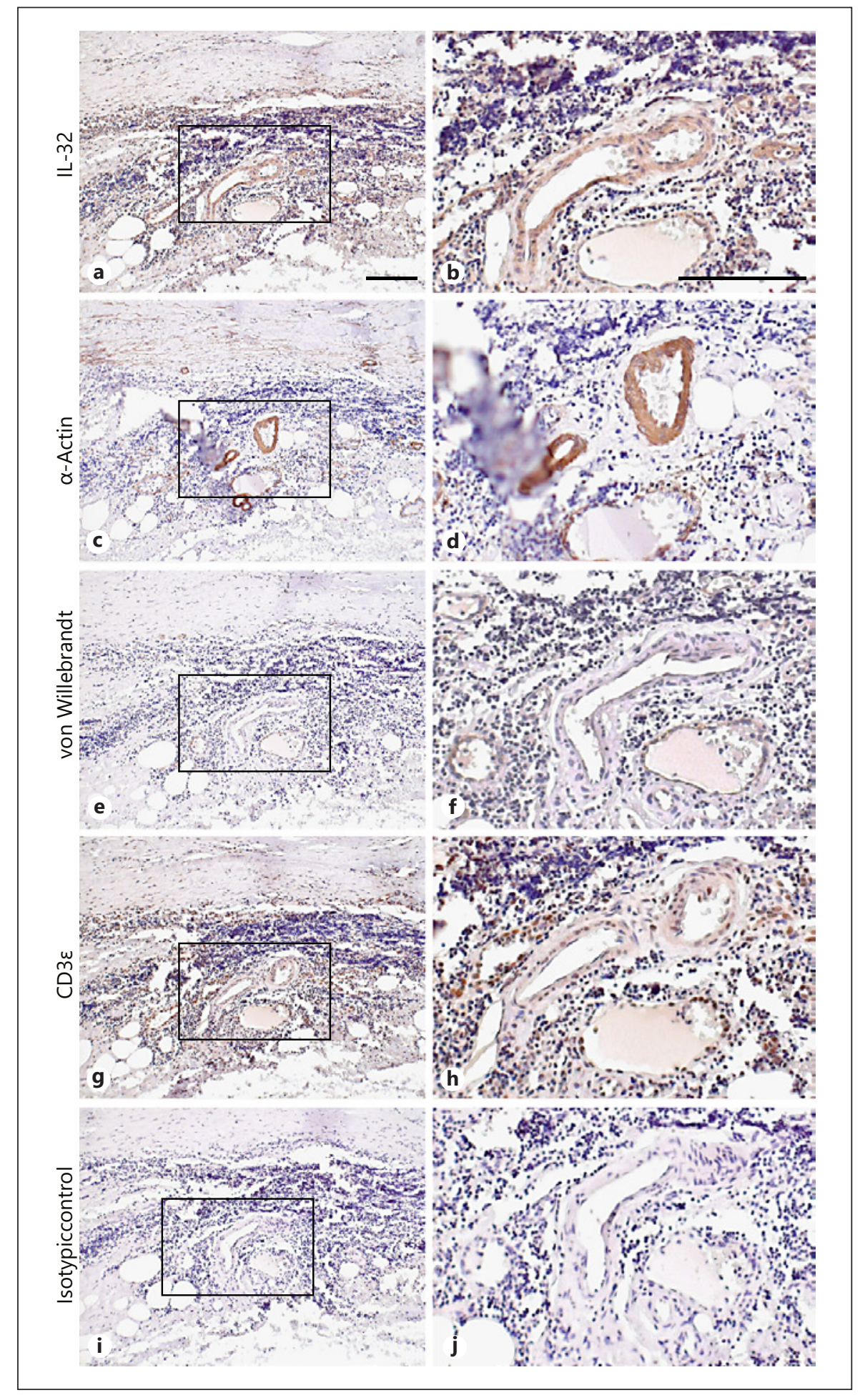

USA), CD3ع (rabbit polyclonal, \#BS6859, Bioworld Technology, St Louis Park, MN, USA), CD68 (mouse monoclonal, \#NCL-CD68KP1, Leica Biosystems, Germany), and von Willebrand factor (rabbit polyclonal, \#A0082, Dako/Agilent, Sweden) were used in serial sections. (See online suppl. Table 3 for antibodies used.) After washing, secondary biotin-conjugated antibodies (goat anti-mouse or goat anti-rabbit, Vector Laboratories, Burlingame, CA, USA) were added for $60 \mathrm{~min}$ at room temperature. Using the avidin-biotin complex solution Vectastain (Vector Laboratories) and diaminobenzidine peroxidase substrate kit (Vector Laboratories), posi- 
Fig. 2. Representative immunohistochemical localization of IL-32 in the tunica intima and media of the aneurysmal tissue from a 65-year-old man with an aneurysm of $60 \mathrm{~mm}$. Expression of IL-32 (a, b), CD3e (c, d), CD66b (e, f), CD68 (g, h), and isotypic IgG control for IL-32 (i, j). Scale bar $=200 \mu \mathrm{m}$. IL-32, interleukin-32.

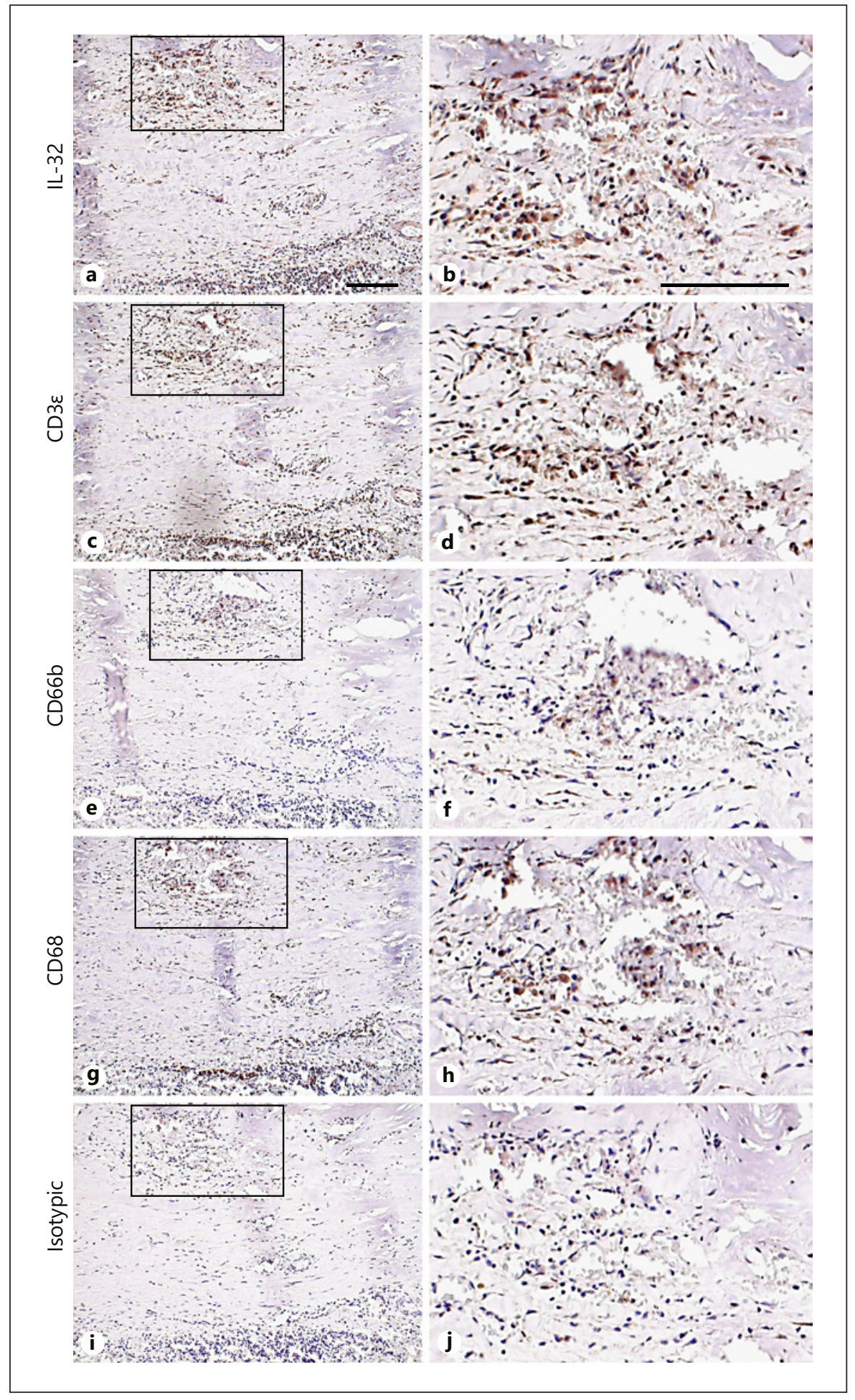

tively stained cells were visualized. Specificity was verified using isotypic controls from the same species (mouse; \#MAB002, R\&D Systems, Inc., Minneapolis, MN, USA, or rabbit; \#NB810-56910, Novus Biologicals, Centennial, CO, USA) and by omitting the secondary antibody as well as using control samples.

Expression of IL-32 in AAA
Western Blot

Using a NuPAGETM 4-12\% Bis-Tris gel (Invitrogen, Carlsbad, CA, USA), samples were separated by electrophoreses set to $180 \mathrm{~V}$ and allowed to run for $45 \mathrm{~min}$ at $+4^{\circ} \mathrm{C}$. The transfer was performed for $60 \mathrm{~min}$ at $+4^{\circ} \mathrm{C}$ set at $100 \mathrm{~V}$ with polyvinylidene difluoride mem- 
Fig. 3. IL-32 expression in aneurysmal aortas. Protein expression of IL-32 in non-aneurysmal control aortas $(n=4,<30 \mathrm{~mm})$ and $\operatorname{AAA}(n=5,70 \pm 2.2 \mathrm{~mm})(\mathbf{a})$. $\beta$-Tubulin used as loading control. Quantification of IL-32 expression (b) by densitometry analysis. Expression of IL-32 mRNA (c) in non-aneurysmal control aortas $(n=9)$, AAA $(n=8)$, and AAA divided into intima/media layer $(n=7)$, adventitia layer $(n=10)$, and PVAT layer $(n=6)$. Statistical analysis of the data from the qPCR of patient samples was performed with an ANOVA and Bonferroni corrections. Determination of the significance of the results of the Western blot was done with Student's $t$ test. Data were considered statistically significant at $p<0.05 .{ }^{*}$ indicates $p$ value $<0.05$. IL-32, interleukin-32; AAA, abdominal aortic aneurysm; PVAT, perivascular aortic tissue.

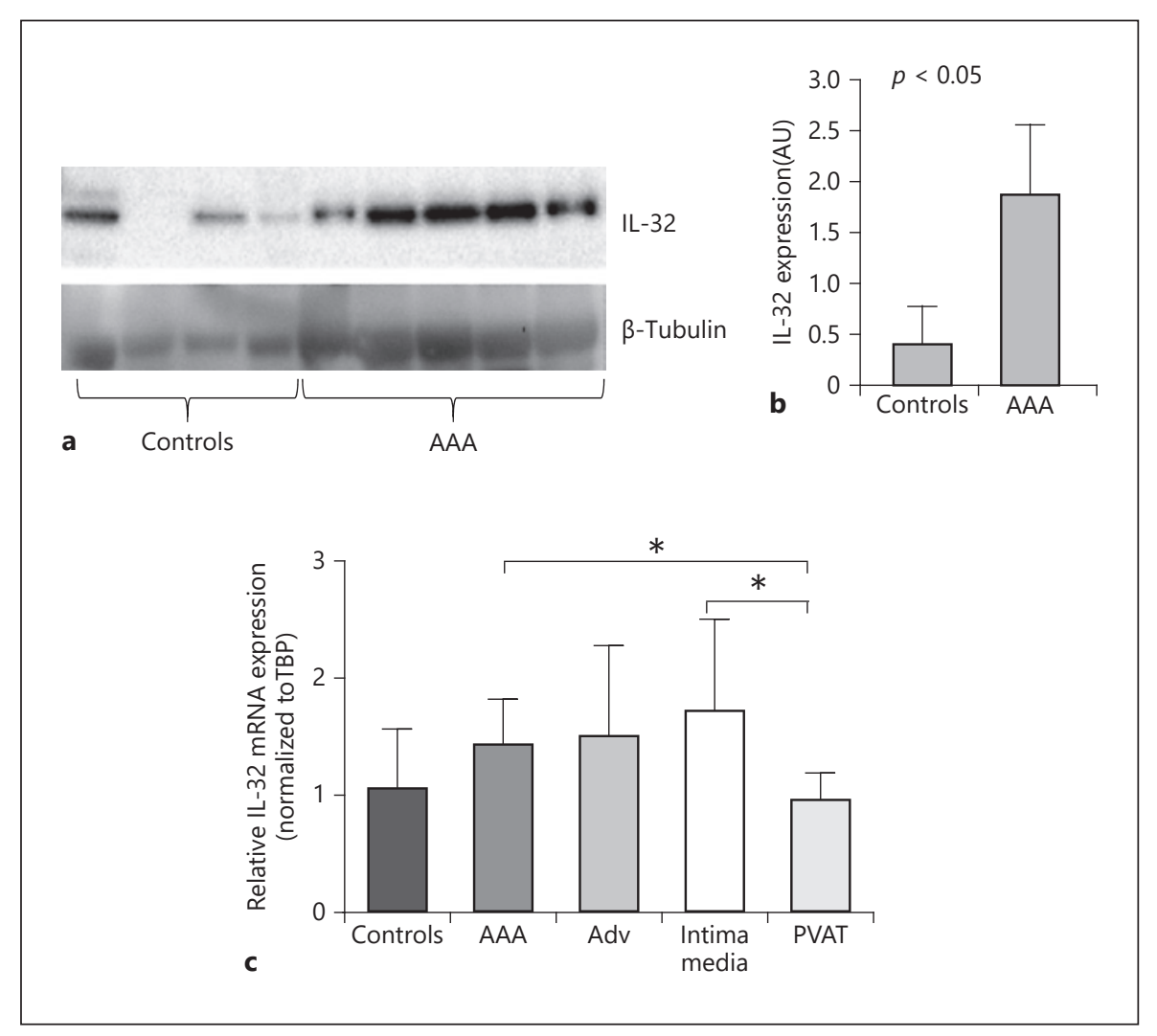

brane (Amersham ${ }^{\mathrm{TM}}$, GE Healthcare, UK). Membranes were incubated overnight at $+4^{\circ} \mathrm{C}$ on a shaker with a primary IL-32 (rabbit polyclonal, Abcam) or $\beta$-tubulin (rabbit polyclonal, \#MA5-16308, Abcam) antibody solution. The list of antibodies is available in online suppl. Table 3. The IL-32 primary antibody was diluted 1:500 (0.002 $\mu \mathrm{g} / \mu \mathrm{L})$ with a fat-free milk solution. The membranes were developed with ECL ${ }^{\mathrm{TM}}$ Prime Western Blotting Detection Reagent (AmershamTM) for $5 \mathrm{~min}$ at room temperature. The image of the membranes was elicited with a gel imager (BioRad, Hercules, CA, USA).

\section{Real-Time $q R T-P C R$}

RNA was isolated from cell culture samples and tissue samples using an RNeasy Mini Kit (Qiagen, Germantown, MD, USA). From the isolated RNA (160 ng), first-strand cDNA was synthesized with the SuperScript ${ }^{\mathrm{TM}}$ III First-Strand Synthesis System (ThermoFisher Scientific, Waltham, MA, USA). cDNA was amplified using TaqMan polymerase (Qiagen) and an exon-overlapping fluorescent IL-32 probe (\#Hs0099241_m1) and TATA box-binding protein probe (for normalization, \# Hs00427620_m1). The amplification was done in a 7500 Fast Real-Time PCR Sequence Detector (Applied Biosystems; Thermo Fisher Scientific, Inc., Waltham, MA, USA) in duplicate.

\section{ELISA}

Circulating levels of IL-32 (designed to detect the isoforms, i.e., $\alpha, \beta$, and $\gamma$ ) were measured in plasma from AAA patients and ageand sex-matched controls using an ELISA kit, according to the manufacturer's protocol (R\&D Systems, Inc.) and as described in our previous study [28].

\section{Statistical Analysis}

Statistical analysis of the data from the qPCR of patient samples was performed with an ANOVA and Bonferroni corrections. Determination of the significance of the results of the Western blot was done with Student's $t$ test. Data were considered statistically significant at $p<0.05$.

\section{Results}

\section{IL-32 Expressed Is Higher in AAA Samples and \\ Localized to SMCs, T Cells, Macrophages, and \\ Endothelial Cells}

Using immunohistochemical analysis, IL-32 protein was predominantly expressed in SMCs in the vasa vasorum and medial SMCs (Fig. 1), T-cell (CD3e)-rich areas in the media and adventitia (Figs. 1 and 2), and macrophage (CD68)-rich areas in the intima/media layer (Fig. 2) in the aneurysmal tissue. IL-32 was expressed in endothelial cells (Fig. 1) in the vasa vasorum, and only weakly or not detected in CD66b-stained neutrophils (Fig. 2). Expression of IL-32 was negative or very weak in non-aneurysmal aortas (online suppl. Fig. 1).

Using Western blot analysis, immunohistochemical results indicating that $\mathrm{IL}-32$ is abundant in AAA were 


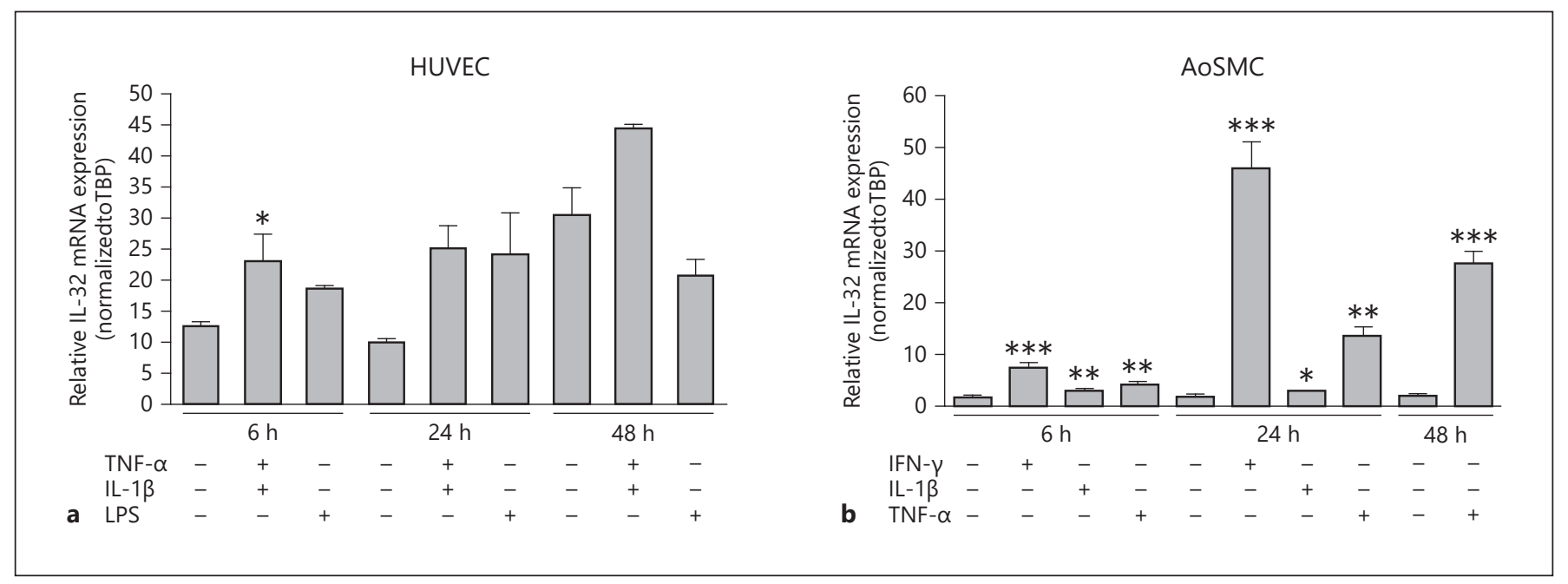

Fig. 4. Expression of IL-32 mRNA in cultured HUVECs (a) and AoSMC (b) from 3 separate experiments. Statistical analysis of the data from the qPCR was performed with an ANOVA and Bonferroni corrections. * indicates $p$ value $<0.05,{ }^{* *} p$ values $<0.01$, and ${ }^{* * *} p$ values $<0.001$, versus respective control and time point. IL, interleukin; HUVEC, human vascular endothelial cell; AoSMC, aortic smooth muscle cell; INF- $\gamma$, interferon- $\gamma$; TNF- $\alpha$, tumor necrosis factor- $\alpha$; LPS, lipopolysaccharide.

confirmed. IL-32 protein (Fig. 3a, b) was expressed significantly higher (4.6-fold) in AAA $(1.9 \pm 0.69$ AU) samples than in non-aneurysmal aortas $(0.4 \pm 0.38 \mathrm{AU}, p<$ $0.05)$.

The presence of IL-32 mRNA was slightly higher in AAA than in control aortas but did not reach significance (Fig. 3c). IL-32 mRNA was detected in the intima/media, adventitia, and PVAT areas of the AAA tissue, with the highest observed average values in the intima/media layers. Levels of IL-32 mRNA were significantly lower in PVAT from AAA aortas than in the intima/media layer and whole AAA aortas that lack PVAT.

IL-32 Is Expressed by Endothelial Cells, SMCs, T Cells, and Monocytes and Induced by Cytokines in vitro

In cultured HUVECs and aortic SMCs, the HUVECs had the highest basal expression, while stimulation of SMCs with TNF- $\alpha$ increased the levels to that of unstimulated HUVECs. IFN- $\gamma$ stimulation of the SMCs leads to even higher expression, similar to TNF- $\alpha$ - and IL-1 $\beta$ stimulated HUVECs (Fig. 4). Although only lipopolysaccharide reached significant induction in HUVECs, cytokine stimulation at 24 and $48 \mathrm{~h}$ was nearly significant ( $p=0.051$ and $p=0.088$, respectively).

To further validate that IL-32 is expressed by T cells and macrophages, cell culture experiments were conducted. Peripheral blood mononuclear cells, that is, neutrophils, monocytes, B cells, and T cells, isolated from 3 healthy individuals showed that $\mathrm{T}$ cells had the highest basal mRNA expression of IL-32, about 10-fold as high as that in the other cell types (Fig. 5). Since IL-32 is inducible by cytokines such as IFN- $\gamma$, the peripheral blood mononuclear cells were stimulated with this cytokine. Monocytes responded significantly to the stimuli in all donors and resulted in a 10-fold induction $(p<0.05)$. Neutrophils, unstimulated as well as stimulated, did not express IL-32 mRNA to detectable levels (data not shown), which is in agreement with our immunohistochemical results. To further investigate whether IL-32 is produced and secreted from the different leukocytes, media from the cells were analysed for IL-32 content using ELISA. Monocytes were the only cell type among the leukocytes that expressed IL-32 $(26 \pm 31.6 \mathrm{pg} / \mathrm{mL})$ after $40 \mathrm{~h}$ of incubation.

\section{No Differences in the Expression of IL-32 in Plasma between AAA and Controls}

Plasma levels of IL-32 were similar in AAA $(20,765$ pg/ $\mathrm{mL}, n=140)$ patients and age-matched controls $(23,968$ $\mathrm{pg} / \mathrm{mL}, n=37)$. There were no significant differences in IL-32 expression in the AAA patients with respect to clinical variables such as age, gender, underlying cardiovascular disease, hypertension, aortic diameter, or growth rate of the aneurysm (data not shown and online suppl. Table 4). 


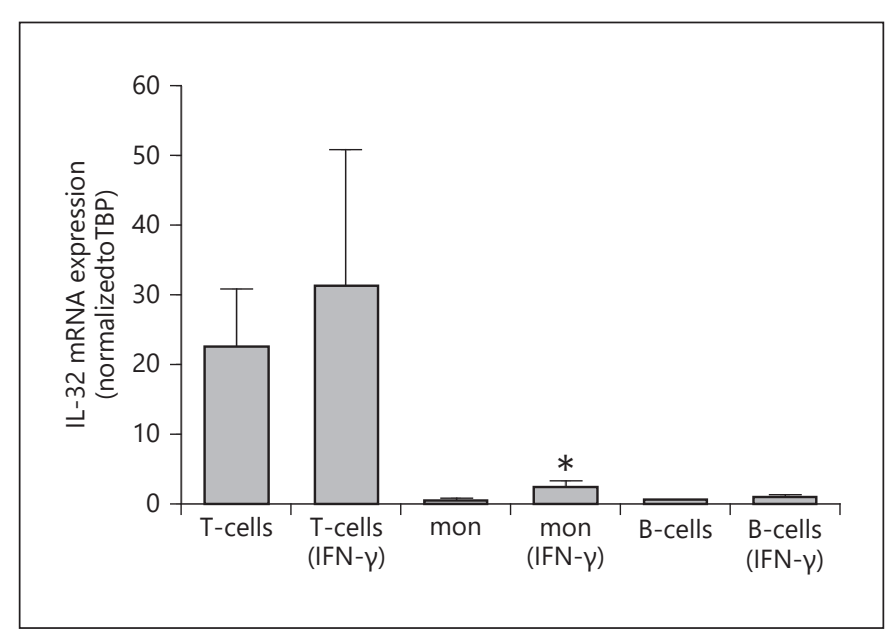

Fig. 5. Expression of IL-32 mRNA in PBMCs, unstimulated or stimulated with IFN- $\gamma$ for $40 \mathrm{~h}$, from 3 donors. Determination of the significance of the results was done with Student's $t$ test. * indicates significant difference between untreated monocytes at $p<0.05$. IL, interleukin; PBMC, peripheral blood mononuclear cell; INF- $\gamma$, interferon- $\gamma$; Mon, monocytes; TBP, TATA box-binding protein.

\section{Discussion}

The aim of this study was to investigate the expression and localization of IL-32 and to identify potential cells capable of producing IL-32 within the tissue of AAA. As of date, no study reported on the relationship of IL-32 with AAA.

When examining AAA tissue with immunostaining, cells within the intima media, that is, SMCs, T cells, endothelial cells, and macrophages, were shown to be expressing IL-32. From the immunohistochemical images of the non-aneurysmal aorta, IL-32 was not expressed within the tissue of the aorta, and immune cells were not present. These two differing images of the vascular walls of an AAA and a non-aneurysmal vessel would suggest that IL-32 signaling and synthesis are altered during or due to the aneurysm pathology. The fact that IL-32 is present in both SMCs and inflammatory cells, T cells predominately, could suggest that IL-32 is not only related to an increase in immune cell accumulation within an AAA but also related to the SMCs' reaction.

The lack of IL-32 expression in non-aneurysmal tissue and that IL-32 was so clearly localized with immune cells, SMCs, and endothelial cells made it interesting to observe how these cell types are affected by pro-inflammatory stimulants in vitro. The fact that these AoSMCs and T cells had an increased expression of IL-32 mRNA after stimulation suggests that the findings in the immunohistochemical images are sound. The expression of mRNA and the presence of IL-32 protein in these cell types suggest that it is within these cell types that the IL-32 signaling is disturbed in AAA. That endothelial cells express IL-32 and is inducible by cytokines is in agreement with previous studies [20,29].

When the levels of IL-32 protein were measured in plasma samples, there were no significant differences in the levels of IL-32 between AAA patients and controls. In a sensitivity analysis, different statistical approaches were used to detect any differences. This could suggest that IL32 has a local effect in AAA tissue. This could also be supported by the fact that when cell cultures were stimulated with the cytokine IL-32, monocytes were the only cells to secrete IL-32 to a detectable level. The fact that there was no significant difference between controls and AAA patients also suggests that IL-32 would not be a suitable protein to be used as a biomarker in clinical practice. There are limited data on whether drugs like statins, aspirin, or other inflammatory cytokines influence IL-32 concentration in plasma. The use of statins or hyperlipidemia among our patients did not influence IL-32 expression in plasma. Li et al. [30] showed in their study that IL-32 induction by influenza A-infected lung epithelial cells is blocked by selective COX-2 inhibitor or aspirin, indicating that IL-32 is induced through COX-2 in the inflammatory cascade. IL-35 is a novel immunomodulatory cytokine produced by regulatory $\mathrm{T}$ cells that induce IL-32 in aortic SMCs and may be a way to regulate immune homoeostasis of the vascular wall [31].

It would be interesting to study the expression of IL-32 in a mouse model of AAA, but due to the lack of a homo$\log$ of IL-32 in mice, and other rodents, it is difficult to find a suitable animal model. However, in a study by Son and colleagues [12], they generated human IL-32 $\alpha$ transgenic mice, making it possible to study the role of human IL-32 in a mouse model of atherosclerosis. Their study showed that IL-32 $\alpha$ inhibited endothelial inflammation, vascular SMC activation, and atherosclerosis by upregulating Timp3 and Reck through suppressing microRNA-205 biogenesis. In addition to these results, in a previous study conducted by us [32], miR-205 was strongly associated with AAA disease. The fact that IL-32 is one of only twenty genes associated with miR-205 and has not been investigated in AAA before makes it interesting to study this pathway closer in future studies.

In conclusion, IL-32 is altered in AAA disease and localized to T cells, macrophages, endothelial cells, and SMCs where it is inducible by pro-inflammatory cytokines. Further studies using transgenic animal models would be helpful to study the potential role of IL-32 in AAA disease. 


\section{Statement of Ethics}

All AAA participants gave written informed consent to the study, which was approved by the regional ethical review board in Linköping, Sweden. The use of organ donors was approved by the regional ethical review board in Lund, Sweden. The study protocol conforms to the ethical guidelines of the 1975 Declaration of Helsinki.

\section{Disclosure Statement}

The authors have no conflicts of interest to declare.

\section{Funding Sources}

This study was supported by grants from the Swedish Heart and Lung Foundation (20190556, D.W.) and the Swedish Research Council (2019-01,673). The founders had no influence on any parts of the study.

\section{Author Contributions}

D.W., M.B., and A.W. conceived the idea; S.B., L.S., D.A., and A.K. performed the experiments; M.W. collected the human material and clinical data; S.B., A.K., and D.W. performed statistical analysis; S.B. and D.W. wrote the manuscript draft; and all authors read through and corrected the manuscript. All authors read and approved the final manuscript.

\section{References}

1 Moll FL, Powell JT, Fraedrich G, Verzini F, Haulon S, Waltham M, et al. Management of abdominal aortic aneurysms clinical practice guidelines of the European society for vascular surgery. Eur J Vasc Endovasc Surg. 2011; 41(Suppl 1):S1-58.

2 Chaikof EL, Dalman RL, Eskandari MK, Jackson BM, Lee WA, Mansour MA, et al. The Society for Vascular Surgery practice guidelines on the care of patients with an abdominal aortic aneurysm. J Vasc Surg. 2018; 67(1):2-77.e2.

3 Beck AW, Sedrakyan A, Mao J, Venermo M, Faizer R, Debus S, et al. Variations in abdominal aortic aneurysm care: a report from the International Consortium of Vascular Registries. Circulation. 2016;134(24):1948-58.

4 Budtz-Lilly J, Björck M, Venermo M, Debus S, Behrendt CA, Altreuther M, et al. Editor's choice: the impact of centralisation and endovascular aneurysm repair on treatment of ruptured abdominal aortic aneurysms based on international registries. Eur J Vasc Endovasc Surg. 2018;56(2):181-8.

5 Björck M, Wanhainen A. Pathophysiology of AAA: heredity vs. environment. Prog Cardiovasc Dis. 2013;56(1):2-6.

6 Abdul-Hussien H, Hanemaaijer R, Kleemann R, Verhaaren BF, van Bockel JH, Lindeman JH. The pathophysiology of abdominal aortic aneurysm growth: corresponding and discordant inflammatory and proteolytic processes in abdominal aortic and popliteal artery aneurysms. J Vasc Surg. 2010; 51(6):1479-87.

7 Kuivaniemi H, Ryer EJ, Elmore JR, Tromp G. Understanding the pathogenesis of abdominal aortic aneurysms. Expert Rev Cardiovasc Ther. 2015;13(9):975-87.

8 Dale MA, Ruhlman MK, Baxter BT. Inflammatory cell phenotypes in AAAs: their role and potential as targets for therapy. Arterioscler Thromb Vasc Biol. 2015;35(8):174655.
9 Busch A, Grimm C, Hartmann E, Paloschi V, Kickuth R, Lengquist $M$, et al. Vessel wall morphology is equivalent for different artery types and localizations of advanced human aneurysms. Histochem Cell Biol. 2017; 148(4):425-33.

10 Ullery BW, Hallett RL, Fleischmann D: Epidemiology and contemporary management of abdominal aortic aneurysms. Abdom Radiol. 2018;43(5):1032-43.

11 Koch AE, Haines GK, Rizzo RJ, Radosevich JA, Pope RM, Robinson PG, et al. Human abdominal aortic aneurysms. Immunophenotypic analysis suggesting an immune-mediated response. Am J Pathol. 1990; 137(5):1199-213.

12 Son DJ, Jung YY, Seo YS, Park H, Lee DH, Kim S, et al. Interleukin-32 $\alpha$ inhibits endothelial inflammation, vascular smooth muscle cell activation, and atherosclerosis by upregulating timp 3 and reck through suppressing microRNA-205 biogenesis. Theranostics. 2017;7(8):2186-203.

13 Dahl CA, Schall RP, He HL, Cairns JS. Identification of a novel gene expressed in activated natural killer cells and T cells. J Immunol. 1992;148(2):597-603.

14 Heinhuis B, Koenders MI, van Riel PL, van de Loo FA, Dinarello CA, Netea MG, et al. Tumour necrosis factor alpha-driven IL-32 expression in rheumatoid arthritis synovial tissue amplifies an inflammatory cascade. Ann Rheum Dis. 2011;70(4):660-7.

15 Kim SH, Han SY, Azam T, Yoon DY, Dinarello CA. Interleukin-32: a cytokine and inducer of TNFalpha. Immunity. 2005; 22(1):131-42.

16 Shoda H, Fujio K, Yamaguchi Y, Okamoto A, Sawada T, Kochi Y, et al. Interactions between IL-32 and tumor necrosis factor alpha contribute to the exacerbation of immuneinflammatory diseases. Arthritis Res Ther. 2006;8(6):R166.
17 Rossi V, Breviario F, Ghezzi P, Dejana E, Mantovani A. Prostacyclin synthesis induced in vascular cells by interleukin-1. Science. 1985;229(4709):174-6.

18 Dinarello CA. Blocking IL-1 in systemic inflammation. J Exp Med. 2005;201(9):1355-9.

19 Voelkel NF, Tuder R. Interleukin-1 receptor antagonist inhibits pulmonary hypertension induced by inflammation. Ann N Y Acad Sci. 1994;725:104-9.

20 Nold-Petry CA, Nold MF, Zepp JA, Kim SH, Voelkel NF, Dinarello CA. IL-32-dependent effects of IL-1beta on endothelial cell functions. Proc Natl Acad Sci USA. 2009; 106(10):3883-8

21 Ribeiro-Dias F, Saar Gomes R, de Lima Silva LL, Dos Santos JC, Joosten LA. Interleukin 32: a novel player in the control of infectious diseases. J Leukoc Biol. 2017;101(1):39-52.

22 Heinhuis B, Popa CD, van Tits BL, Kim SH, Zeeuwen PL, van den Berg WB, et al. Towards a role of interleukin-32 in atherosclerosis. Cytokine. 2013;64(1):433-40.

23 Kobayashi H, Huang J, Ye F, Shyr Y, Blackwell TS, Lin PC. Interleukin-32 $\beta$ propagates vascular inflammation and exacerbates sepsis in a mouse model. PloS One. 2010; 5(3):e9458

24 Vorkapic E, Folkesson M, Magnell K, Bohlooly-Y M, Länne T, Wågsäter D. ADAMTS-1 in abdominal aortic aneurysm. PloS One. 2017;12(6):e0178729.

25 Wågsäter D, Björk $\mathrm{H}$, Zhu C, Björkegren J, Valen G, Hamsten A, et al. ADAMTS-4 and -8 are inflammatory regulated enzymes expressed in macrophage-rich areas of human atherosclerotic plaques. Atherosclerosis. 2008;196(2):514-22.

26 Olofsson PS, Söderström LA, Wågsäter D, Sheikine Y, Ocaya P, Lang F, et al. CD137 is expressed in human atherosclerosis and promotes development of plaque inflammation in hypercholesterolemic mice. Circulation. 2008;117(10):1292-301. 
27 Jatta K, Wågsäter D, Norgren L, Stenberg B, Sirsjö A. Lipopolysaccharide-induced cytokine and chemokine expression in human carotid lesions. J Vasc Res. 2005;42(3):26671.

28 Shamoun L, Kolodziej B, Andersson RE, Dimberg J. Protein expression and genetic variation of IL32 and association with colorectal cancer in Swedish patients. Anticancer Res. 2018;38(1):321-8.
29 Kobayashi H, Lin PC. Molecular characterization of IL-32 in human endothelial cells. Cytokine. 2009;46(3):351-8.

30 Li W, Liu Y, Mukhtar MM, Gong R, Pan Y, Rasool ST, et al. Activation of interleukin-32 pro-inflammatory pathway in response to influenza A virus infection. PloS One. 2008; 3(4):e1985.

31 Skowron W, Zemanek K, Wojdan K, Gorzelak P, Borowiec M, Broncel M, et al. The effect of interleukin-35 on the integrity, ICAM-1 expression and apoptosis of human aortic smooth muscle cells. Pharmacol Rep. 2015;67(2):376-81.

32 Wanhainen A, Mani K, Vorkapic E, De Basso R, Björck M, Länne T, et al. Screening of circulating microRNA biomarkers for prevalence of abdominal aortic aneurysm and aneurysm growth. Atherosclerosis. 2017; 256:82-8 